14th AIAA/CEAS Aeroacoustics Conference, Vancouver, Canada, 2008 Jet Aeroacoustics

\title{
Jet noise from instability mode interactions
}

\author{
Neil D. Sandham*, Adriana M. Salgado ${ }^{\dagger}$ and Anurag Agarwal ${ }^{\ddagger}$ \\ University of Southampton, Southampton SO17 1BJ, U.K.
}

\begin{abstract}
A nonlinear interaction model is used to study sources of sound in jets. The model uses quadratic interactions of instability modes, which are obtained by solving the linear parabolized stability equations. Source terms involving nonlinear interactions are evaluated and a linear wave equation is solved with direct injection of the source terms. Thus the complete method solves only linear partial differential equations, coupled by nonlinear source terms. It allows the contribution of each modal interaction to be studied separately, giving a breakdown of the radiation pattern of each interaction. The method is demonstrated using a fixed base flow matched to the experiment of Stromberg et al. (J. Fluid Mech. 72(2), 1980). The squared streamwise velocity quadrupole is the largest source term for axisymmetric mode interactions, while for helical-helical mode interactions both the squared radial and squared azimuthal velocities are the main contributing sources, despite a strong cancelation effect between them. Results are also presented for an alternative implementation, in which the base flow is allowed to vary according to the Reynolds stresses of the developing instability modes. This model demonstrates sound production during mode growth and subsequent saturation.
\end{abstract}

\section{Introduction}

Subsonic and supersonic jets are known to have significantly different mechanisms of generation of sound. In supersonic jets, the primary flow instability is able to radiate sound effectively, while subsonic jet noise is less tractable to theory (Tam \& Morris, 1980; Tam \& Burton, 1984). There is a body of experimental work that suggests that instability modes (solutions of the compressible Orr-Sommerfeld equation for example) are still present in subsonic jets and may still be contributing to the overall sound radiation, albeit not in as obvious a way as at high speeds. The recent work of Suzuki \& Colonius (2006) has shown that instability waves are present, even in turbulent jets at Reynolds numbers up to $10^{6}$. The role of instability waves is naturally clearest in work at lower Reynolds numbers. Laufer \& Yen (1983) located the source of sound with the nonlinear saturation of instability waves and noted that, while the near field pressure varied linearly with disturbance amplitude, the acoustic far field had a quadratic dependence. Stromberg et al. (1980) made experiments at jet Mach number $M=0.9$ and $R e=3600$ based on the jet diameter and also suggested a nonlinear mechanism of noise generation. The Stromberg et al. experiment is attractive for analysis and simulation, not least because the authors included velocity profiles from the region near the nozzle exit. This case was the subject of a direct numerical simulation study by Freund (2001) in which good agreement was found with the experiment.

A widely-used model for sound generation in shear flows is the Lilley-Goldstein equation (Lilley 1974; Goldstein 2001), which extends Lighthill's approach (Lighthill 1952; Morfey \& Wright 2007) to be applicable to disturbances on a parallel shear flow. Retaining only the highest order source terms, the Lilley-Goldstein equation can be written in cylindrical polar co-ordinates with radial direction $r$ and axial direction $z$ as

$$
\frac{\bar{D}}{\bar{D} t}\left(\nabla^{2} \pi+\frac{\bar{D}}{\bar{D} t}\left(\frac{1}{\bar{c}^{2}}\left(q-\frac{\bar{D} \pi}{\bar{D} t}\right)\right)-\frac{1}{\bar{\rho}} \frac{\partial \bar{\rho}}{\partial r} \frac{\partial \pi}{\partial r}-\bar{\rho} \nabla \cdot \vec{f}\right)+2 \frac{\partial \bar{V}_{z}}{\partial r}\left(\bar{\rho} \frac{\partial f_{r}}{\partial z}-\frac{\partial^{2} \pi}{\partial z \partial r}\right)=0,
$$

*Professor, School of Engineering Sciences, n.sandham@soton.ac.uk, AIAA Senior Member

$\dagger$ PhD Student, ISVR

$\ddagger$ Lecturer, ISVR 
where $\bar{D}=\partial / \partial t+\bar{V}_{z} \partial / \partial z, \pi$ is the wave variable and the parallel shear flow is defined by the axial velocity $\bar{V}_{z}(r)$, density $\bar{\rho}(r)$ and sound speed $\bar{c}(r)$. At highest order for approximately isothermal jets the source terms are given by

$$
q=0 \quad \vec{f}=-\nabla \cdot(\vec{u} \vec{u}) .
$$

The quadratic source term will later be written as a product of instability modes.

The Lilley-Goldstein formulation is applicable to parallel shear flows and for a growing round jet it is not clear a priori which axial location to use in fixing the parallel flow; results are certainly sensitive to this choice, as shown later. If the base flow terms are dropped completely from (1) we recover a simpler form of the equation as

$$
\frac{1}{c_{0}^{2}} \frac{\partial^{2} \pi}{\partial t^{2}}-\nabla^{2} \pi=\rho_{0} \nabla \cdot(\nabla \cdot(\vec{u} \vec{u}))
$$

where $c_{0}$ and $\rho_{0}$ are respectively the sound speed and density of the quiescent fluid into which the sound radiates. Here, the left hand side is a standard second order wave equation and the right hand side contains the source terms involving the products of velocity fluctuations that are the most important sources in jets in the absence of strong temperature variations.

The present work is a continuation of a line of research into the relevance of nonlinear mode interactions to sound radiation that we have been pursuing recently. In Sandham et al. (2006a) a model problem was used to show how products of linear instability modes could be used as source terms, leading to potentially useful predictions of the main characteristics of the sound field. Any quadratic mode interaction can be split into a term involving the sum of the wavenumbers (or frequencies in the spatial case) and a term involving the differences. In a companion paper Sandham et al. (2006b) showed that wave packets based on growing and decaying envelope functions are inefficient at radiating sound for low Mach numbers, for slow saturation time scales and for high frequencies, hence interactions involving the summed wavenumbers will be less effective radiators than those involving difference wavenumbers. This was confirmed for the plane jet model problem, where the difference in wavenumber of two instability modes gave the longer wavelength of the radiated sound field compared to the primary instability wavelength, in accordance with numerical solutions of the compressible Navier-Stokes equations. Figure 1 sketches how this idea is applied to the single stream round jet considered in this paper. The potential core disappears where the annular mixing layers merge. Two spatially-developing unstable waves inside the jet with different frequencies $f_{1}$ and $f_{2}$ may interact via the source term in (1) to give effective sound radiation with frequency $f_{1}-f_{2}$.

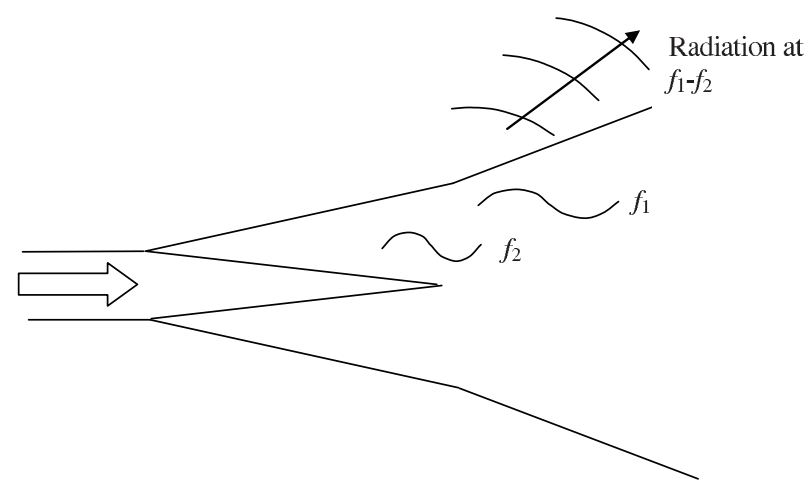

Figure 1. Schematic of the round jet problem, showing how interactions between instability waves within the jet can drive a difference mode in the acoustic field.

Sandham and Salgado (2008) extended the method to spatially-developing waves, and calculations were made using the stability eigenvalues as forcing on the surface of a cylinder. The method was shown to give the Strouhal number of the jet as $S t=0.45$ and of the sound field as $S t=0.19$, in good agreement with the experiments of Stromberg et al (1980), which gave 0.44 and 0.20 respectively. In the present contribution we make use of the complete mode shapes by injecting the interaction terms separately into a wave equation solver. This method allows us to find the most important source terms as well as the most important stability mode interactions. We also consider how the specification of a fixed base flow may be avoided, by using the Reynolds stresses of the stability mode interactions to determine the base flow growth in the downstream 
direction. This latter part of the work continues a line of research explored initially by Morris et al. (1989), where stability theory was used to explain certain features of compressible mixing layers.

\section{Formulation}

\section{A. Nonlinear interaction model}

In cylindrical co-ordinates with $z$ the streamwise direction, $r$ the radial direction and $\theta$ the azimuthal direction, we consider the interaction of two spatially-developing instability modes with different (real) frequencies $\omega$ and azimuthal wavenumbers $n$, and an evolution given by

$$
u_{j k}=\hat{u}_{j k} e^{i\left(n_{k} \theta-\omega_{j} t\right)} e^{\int\left(\sigma_{j k}+i \alpha_{j k}\right) d z}+c . c .
$$

and

$$
u_{l m}=\hat{u}_{l m} e^{i\left(n_{m} \theta-\omega_{l} t\right)} e^{\int\left(\sigma_{l m}+i \alpha_{l m}\right) d z}+c . c .
$$

Here c.c. denotes complex conjugate, $\hat{u}(r)$ is a complex eigenfunction and the complex wavenumber has been split into a real part $\alpha$ and an imaginary part $-\sigma$, both of which may vary in the $z$ direction. Subscripts $j$ and $k$ relate the frequency and azimuthal wavenumber of the first mode, while subscripts $l$ and $m$ relate to the respective properties of the second mode. By direct multiplication, it is possible to write down explicitly the source terms for the wave equation, for example

$$
\frac{\partial^{2} u_{j k} u_{l m}}{\partial z^{2}}=\hat{u}_{j k} \hat{u}_{l m} A_{j k l m}^{+} e^{i\left[\left(n_{k}+n_{m}\right) \theta-\left(\omega_{j}+\omega_{l}\right) t\right]}+\hat{u}_{j k} \hat{u}_{l m}^{*} A_{j k l m}^{-} e^{i\left[\left(n_{k}+n_{m}\right) \theta-\left(\omega_{j}-\omega_{l}\right) t\right]}+c . c .
$$

where $^{*}$ denotes complex conjugate and $A^{+}$and $A^{-}$are sum and difference mode amplitudes, given by

$$
A_{j k l m}^{+}=\left[\sigma_{j k}+\sigma_{l m}+i\left(\alpha_{j k}+\alpha_{l m}\right)\right]^{2} e^{\int\left[\sigma_{j k}+\sigma_{l m}+i\left(\alpha_{j k}+\alpha_{l m}\right)\right] d z}
$$

and

$$
A_{j k l m}^{-}=\left[\sigma_{j k}+\sigma_{l m}+i\left(\alpha_{j k}-\alpha_{l m}\right)\right]^{2} e^{\int\left[\sigma_{j k}+\sigma_{l m}+i\left(\alpha_{j k}-\alpha_{l m}\right)\right] d z} .
$$

It can be seen that in both cases the growth rates $\sigma$ add, while the wavenumbers can either add or subtract. A basic radiation pattern for any mode interaction can be found by solving a wave equation forced by (6), as was done by Sandham \& Salgado (2008).

\section{B. Parabolized Stability Equations}

The mode properties $\alpha, \sigma$ and $\hat{u}$ are found by solving the parabolized stability equations (PSE). The PSE method (Herbert, 1997) is an efficient way of generating mode shapes and wavenumbers in convectively unstable flows. With PSE it is also straightforward to incorporate non-parallel and disturbance evolution effects that would not be included in the simpler linear stability approach based on parallel flow. The PSE system of equations can be written in concise notation as

$$
\mathbf{M} \frac{d \hat{\phi}}{d z}=(\omega \mathbf{K}-\mathbf{L}) \hat{\phi}
$$

where $\mathbf{K}, \mathbf{L}$ and $\mathbf{M}$ are matrices and $\hat{\phi}$ is a vector of mode shapes $\left(\hat{\rho}, \hat{v}_{r}, \hat{v}_{\theta}, \hat{v}_{z}, \hat{T}\right)$. The matrix $\mathbf{L}$ contains derivatives in the $r$ direction which are computed using a sixth order compact difference scheme on a stretched mesh, extending to $r=L_{r}$ in the radial direction. The contents of the matrices can be found for example in Hein (2005). PSE has recently been applied to jet noise by Cheung et al. (2007). Linear PSE was found to be able to predict noise from supersonic jets, while nonlinear PSE, together with an acoustic analogy was needed for subsonic jet noise. In the present work, by contrast, we use only linear PSE and compute the nonlinear interaction terms separately.

The solution starts at $z=0$ with a solution for $\hat{\phi}, \sigma$ and $\alpha$ from the compressible Orr-Sommerfeld equation for a given $\omega$. The Orr-Sommerfeld problem was solved with the same compact finite difference scheme in $r$ and used a direct matrix eigenvalue method to find all the eigenvalues and eigenvectors, from which the most unstable mode was selected. After premultiplying by $\mathbf{M}^{-1}$, equation (7) is advanced in $z$ using first order backward differences. Following the standard PSE implementation, at each step the wavenumber $\alpha-i \sigma$ is adjusted iteratively so that

$$
\int_{0}^{L_{r}} \hat{\phi}^{*} \frac{\partial \hat{\phi}}{\partial z} r d r=0
$$




\section{Base flow velocity profiles}

An analytic velocity profile was matched to the available experimental data of Stromberg et al.(1980). The profile gives the axial velocity $\bar{V}_{z}$ at any $z$ and $r$ location using

$$
\bar{V}_{z}=0.5\left[\tanh \left(\frac{r+a}{\delta}\right)-\tanh \left(\frac{r-a}{\delta}\right)\right],
$$

with empirically-determined coefficients

$$
a=0.59+0.09 \tanh (\sqrt{z}-2.9)
$$

and

$$
\delta=\frac{39+24 z+0.11 z^{4}}{1000+z^{3}} .
$$

Examples of the resulting velocity profiles are shown on Figure 2. Profiles of the radial component of velocity $\bar{V}_{r}$ are computed using the steady continuity equation. The jet is swirl-free, so $\bar{V}_{\theta}=0$. For this base flow the pressure is assumed to be uniform and the temperature is approximated by means of a Crocco-Busemann relation

$$
\bar{T}=1+\frac{\gamma-1}{2} M^{2} \bar{V}_{z}\left(1-\bar{V}_{z}\right),
$$

taking the jet temperature equal to the ambient temperature. The jet Reynolds number was set to $R e=3600$ and the jet Mach number to $M=0.9$ to match the conditions of Stromberg et al. and Freund (2001).

The PSE calculations continued downstream to $z=20$. The box size was chosen to be $L_{r}=30$ with 80 points in $r$ and 81 points in $z$. A strong stretching of the grid was used in the radial direction to concentrate points in the jet shear layer.

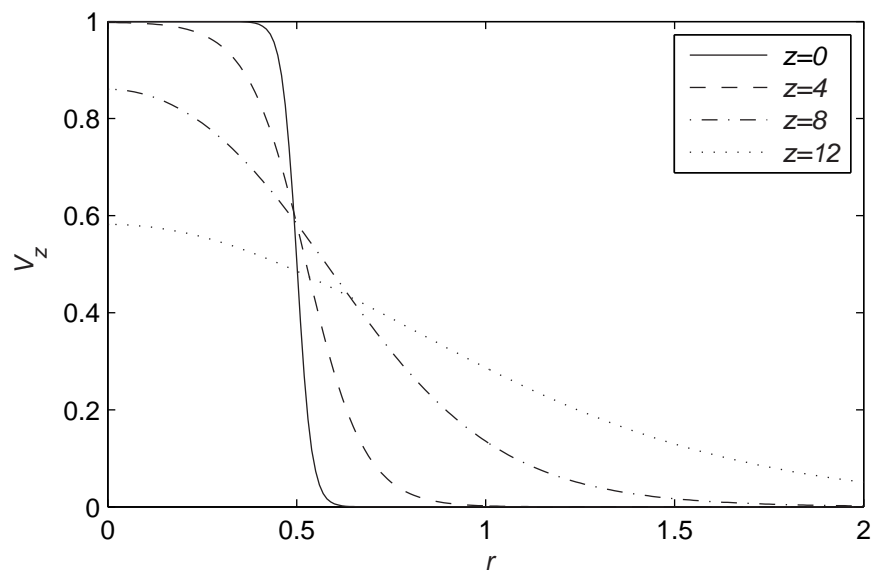

Figure 2. Profiles of the streamwise velocity $\bar{V}_{z}$ at various $z$ locations.

\section{Acoustic source terms}

Initially we consider solutions of equation (3), i.e. the simplified second order wave equation. Later we will make some comparisons with results found by solving the full form of equation (1), but with a simplified forcing scheme. For computational convenience the wave equation is split into a set of first order equations and are solved in the time domain using a standard fourth order Runge-Kutta method.

The source terms in the divergence term $\nabla \cdot \vec{f}$ in (1) can be decomposed into nine terms, defined in 
cylindrical polar co-ordinates as

$$
\begin{array}{ccc}
S_{r r}=\frac{1}{r} \frac{\partial^{2}\left(r u_{r} v_{r}\right)}{\partial r^{2}} & S_{r \theta}=\frac{1}{r^{2}} \frac{\partial^{2}\left(r u_{r} v_{\theta}\right)}{\partial r \partial \theta} & S_{r z}=\frac{1}{r} \frac{\partial^{2}\left(r u_{r} v_{z}\right)}{\partial r \partial z} \\
S_{\theta r}=\frac{1}{r^{2}} \frac{\partial^{2}\left(r u_{\theta} v_{r}\right)}{\partial \theta \partial r} & S_{\theta \theta}=\frac{1}{r^{2}} \frac{\partial^{2}\left(u_{\theta} v_{\theta}\right)}{\partial \theta^{2}}-\frac{1}{r} \frac{\partial\left(u_{\theta} v_{\theta}\right)}{\partial r} & S_{\theta z}=\frac{1}{r} \frac{\partial^{2}\left(u_{\theta} v_{z}\right)}{\partial \theta \partial z} \\
S_{z r}=\frac{1}{r} \frac{\partial^{2}\left(r u_{z} v_{r}\right)}{\partial z \partial r} & S_{z \theta}=\frac{1}{r} \frac{\partial^{2}\left(u_{z} v_{\theta}\right)}{\partial z \partial \theta} & S_{z z}=\frac{\partial^{2}\left(u_{z} v_{z}\right)}{\partial z^{2}}
\end{array}
$$

Other source terms are present in $\partial f_{r} / \partial z$ but, for reasons discussed later, these are not considered in the present paper. The source terms are computed from the PSE modes, including derivatives of mode shapes, and are then interpolated onto a fine grid for solution of the wave equation. The wave equation is Fourier transformed in the azimuthal direction to reduce it to a two-dimensional problem for each specified azimuthal difference mode. Since the initial aim was only to study the jet near sound field, solutions were computed using simple second order central differences on a grid with $401 \times 301$ grid points in the streamwise and radial directions respectively and were run to a specified time in a computational domain twice as large as shown in the figures, such that disturbances had no time to reflect (however weakly) from the boundaries and affect the computed solution. No filtering or dissipation was applied and for stability reasons, in contrast to the PSE calculations, only a weak grid stretching in the radial direction could be used. Boundary conditions are applied at the origin separately for axisymmetric and azimuthal disturbances according to the analysis of Lewis \& Bellan (1990). Thus boundary conditions are specified as $\partial \pi / \partial r=0$ on $r=0$ for $\Delta n=0$ or as $\pi=0$ for $\Delta n \neq 0$, where $\Delta n$ is the difference azimuthal wavenumber.

\section{Results for a fixed base flow}

\section{A. Axisymmetric mode interactions using simple wave equation}

To begin the discussion of results from the interaction model, we consider axisymmetric $\left(n_{k}=0, n_{m}=0\right)$ mode interactions and take as an example the interaction between $\omega_{j}=3.4$ and $\omega_{l}=2.2$, giving a difference mode Strouhal number of 0.19. This interaction is close to the maximum $\left|A^{-}\right|$found by Sandham \& Salgado (2008). Figure 3(a) shows the sound field resulting from forcing separately with $S_{r r}, S_{r z}, S_{z r}$ and $S_{z z}$. The same contour levels are used in each case and it should be noted that, since the base flow for the linear stability analysis is fixed, it is not possible to say anything about the absolute levels of sound radiation. It can be seen that the largest sound is produced by the $S_{r r}$ and $S_{z z}$ quadrupoles. Taking the source origin as $(r=0, z=5)$ the $S_{r r}$ quadrupole radiates preferentially at an angle of $60^{\circ}$ to the downstream direction, while the $S_{z z}$ quadrupole radiates predominantly in the downstream direction. The cross terms involving products of radial and longitudinal velocity are small in comparison. It should be noted that $S_{r z} \neq S_{z r}$ due to the different frequencies involved in the interactions.

In figure $3(\mathrm{~b})$ we show the source distributions corresponding to the sound radiation patterns shown on figure 3(a), taking a reference time of $t=0$. The same contour levels are chosen in each case, but to show the structure more clearly the $S_{r r}$ source has been divided by a factor of four, while the $S_{z z}$ source has been multiplied by a factor of two. This immediately demonstrates the perils of trying to correlate source strength with sound radiation; the $S_{z z}$ quadupole has the weakest source field but radiates the strongest sound. The structure of the source is evidently more important in determining the radiation characteristic than the amplitude.

For the same case, we can solve for the sum modes, giving a Strouhal number of 0.89. Results are shown on Figure 4, using the same contour levels for comparison with the difference modes shown on figure 3(a). Clearly the higher frequencies radiate much less effectively than low frequencies and only the source region is visible on this plot. In most of the discussion that follows we will only be referring to difference mode interactions and focusing on the low-Strouhal number $(S t \approx 0.2)$ range of sound radiation. The relevance, if any, of the stability modes to higher frequency sound remains to be explored; it is possible that higher harmonics of individual modes (i.e. the nonlinear structure of the rolled up vortices) or indeed the fine scale structure of turbulence, may be more important than the summed instability mode interaction terms. 

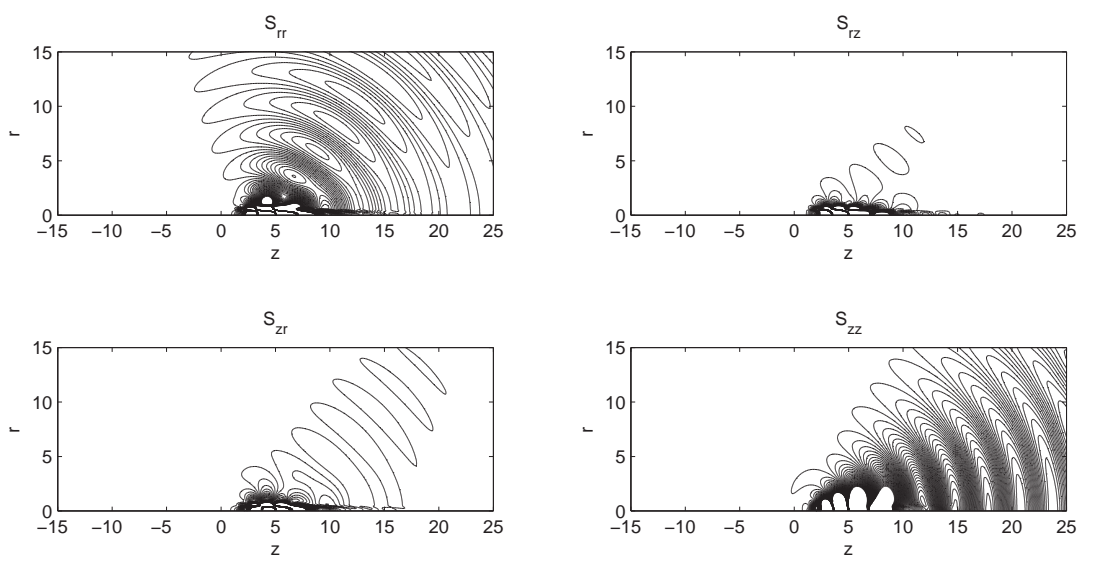

(a)
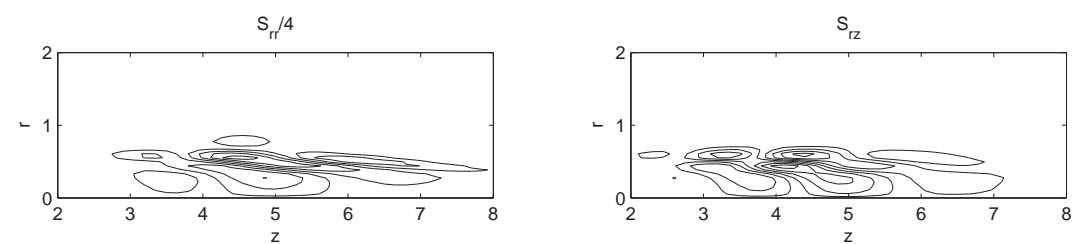

$\mathrm{S}_{\mathrm{zr}}$

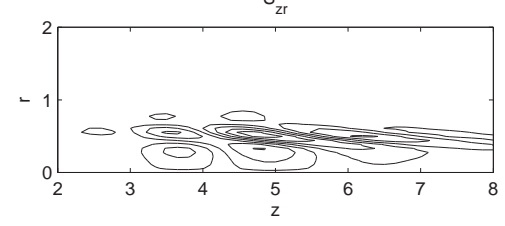

$2 \mathrm{~S}_{\mathrm{zz}}$

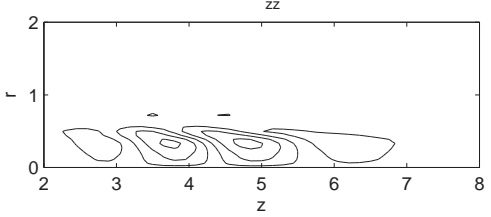

(b)

Figure 3. Solution of wave equation driven by the difference mode interaction of axisymmetric modes: (a) pressure field $\pi$ arising from each source term as shown in the sub-figure title, and (b) the corresponding source field. Note that the $S_{r r}$ source term has been divided by a factor of 4 and the $S_{z z}$ term has been multiplied by a factor of 2 so that the structure can be more clearly seen (otherwise contour levels in the sub-figures are the same).
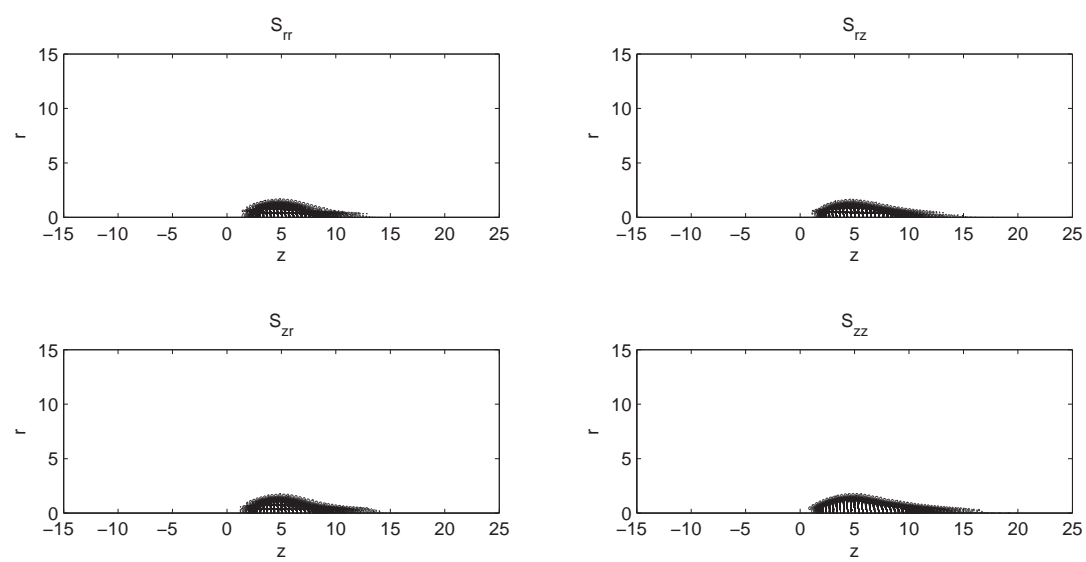

Figure 4. Pressure contours from solution of wave equation driven by the interaction of the sum of two axisymmetric modes. Source terms are injected for $S_{r r}, S_{r z}, S_{z r}$ and $S_{z z}$. 


\section{B. Helical mode interactions}

In the $M=0.9$ jet helical modes are at least as important as axisymmetric instability modes. According to stability theory the $n=1$ mode grows to amplitudes comparable to those found for $n=0$ while higher helical modes are less unstable. Stromberg et al (1980) found that at $S t=0.44$ (the spectral peak found by a hot wire inside the jet) both $n=0$ and $n=1$ components were present, while at $S t=0.22$ (the spectral peak of the sound radiation) the flow was predominantly axisymmetric (with no significant phase change around the jet azimuth).

Figures 5 and 6 show respectively the $(0,1)$ and $(1,0)$ mode interactions that lead to $\Delta n=1$ radiation from the jet. All the non-zero sources are shown using the same contour levels as used for Figure 3(a). The frequencies of the modes were chosen as $\omega_{j}=3.2$ and $\omega_{l}=1.8$ (for maximum $\left|A^{-}\right|$) giving a difference mode $S t=0.22$. Again due to the different frequencies there is no symmetry between the $(0,1)$ and $(1,0)$ interactions. The largest radiation comes from the $S_{z \theta}$ quadrupole, but the sound production is lower than for the axisymmetric $(0,0)$ interaction. The relative amplitude of the radiation is frequency dependent and has not been fully explored since, according to the model, very small difference frequencies give rise to very high radiation levels, which are not believed to be physical. Nevertheless, using the largest $\left|A^{-}\right|$for each mode pair gives a result that agrees with the experimental finding that the sound is mainly axisymmetric for this jet configuration.

Axisymmetric sound radiation also arises from the $(1,1)$ interaction shown on Figure 7 . Here we take $\omega_{j}=3.0$ and $\omega_{l}=1.8$ (for maximum $\left|A^{-}\right|$) giving a difference mode $S t=0.19$. In this case extremely large levels of radiation are seen to arise from the $S_{r r}$ and $S_{\theta \theta}$ quadrupoles when considered separately. However these two sound fields are close to being in anti-phase and when the sum of the two is plotted in the last sub-figure (lower right) it can be seen that the overall radiation is at a much lower level (and the overall sound radiation is slightly lower than that observed for the $(0,0)$ interaction).
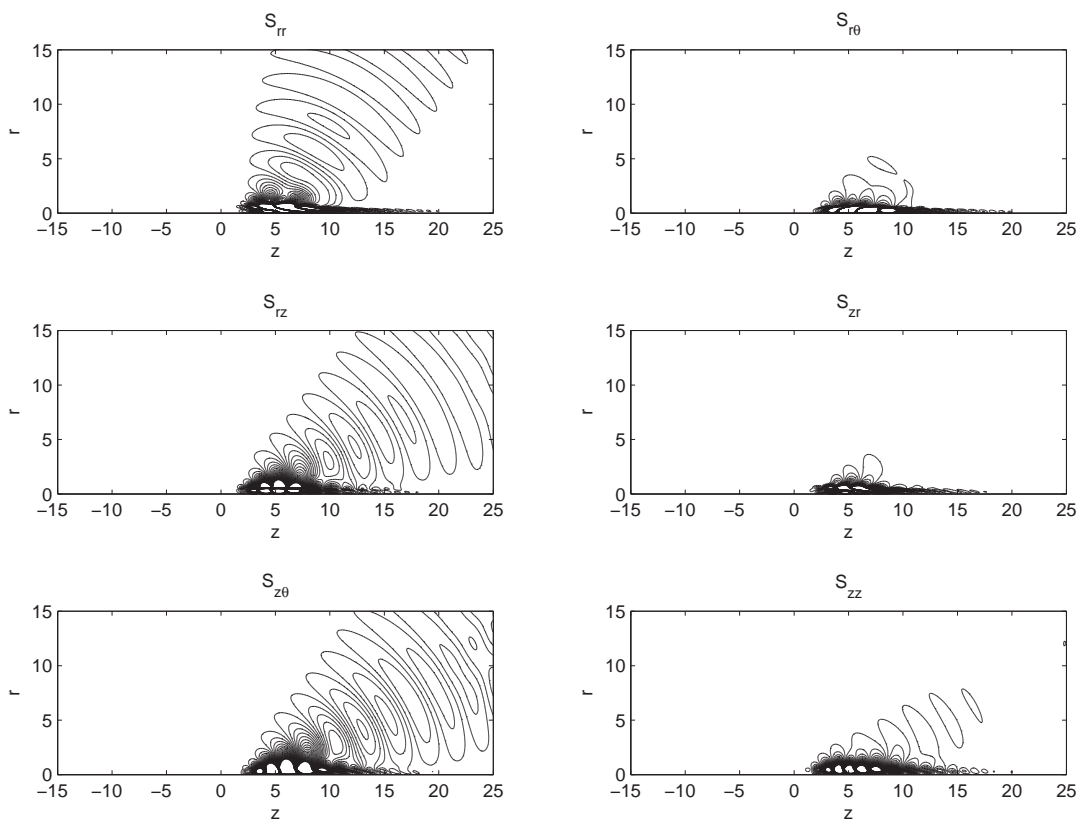

Figure 5. Pressure contours from the (0-1) interaction of a axisymmetric mode (at higher frequency) with a helical mode (at lower frequency). 

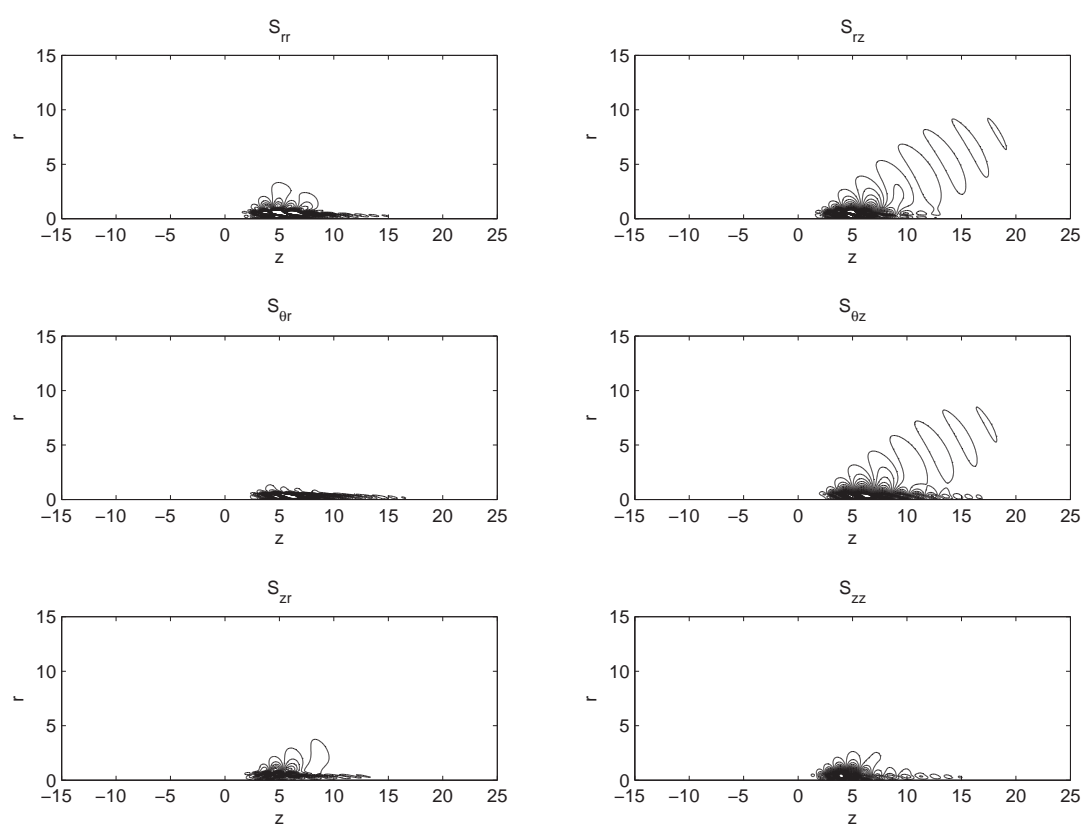

Figure 6. Pressure contours from the (1-0) interaction of a helical mode (at higher frequency) with an axisymmetric mode (at lower frequency).
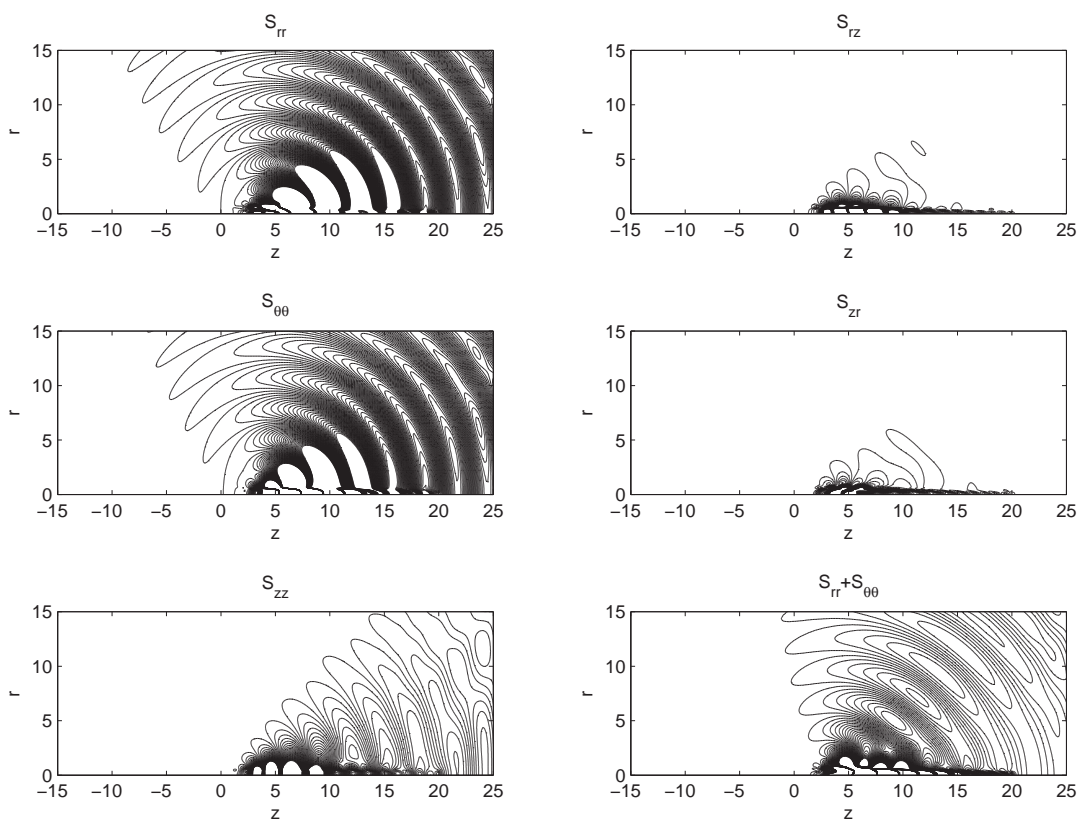

Figure 7. Pressure contours from the (1-1) difference interaction of two helical modes. The lower right hand sub-figure shows $S_{r r}+S_{\theta \theta}$ and illustrates the large cancelation that is occurring between these components. 
(a)
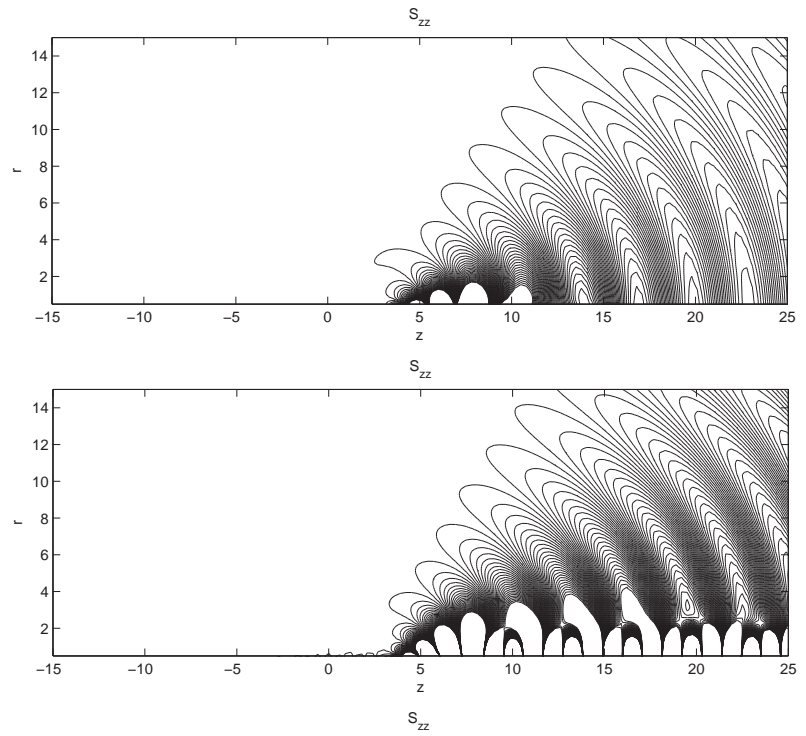

(b)

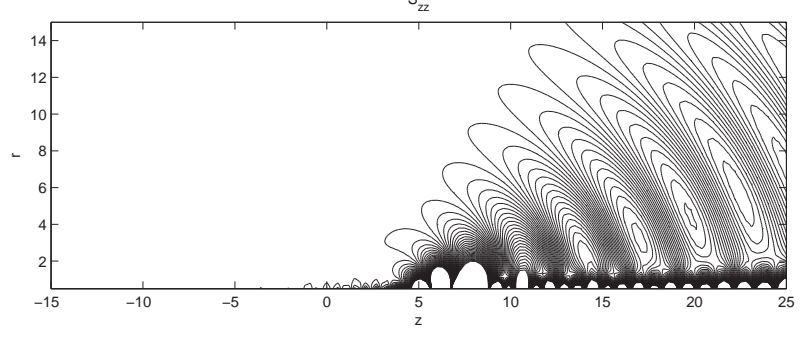

(c)

Figure 8. Sound from source $S_{z z}$ solving the Lilley-Goldstein equation with forcing on $r=r_{0}$ : (a) $\bar{V}_{z}=0$ and $\bar{\rho}=1$, (b) $\bar{V}_{z}$ and $\bar{\rho}$ taken at $z_{0}=5.0$ and (c) $\bar{V}_{z}$ and $\bar{\rho}$ taken at $z_{0}=15.0$.

\section{Comparison with Lilley-Goldstein formulation}

The Lilley-Goldstein equation (1) represents potentially a more realistic description of the sound radiation since it separates sources from propagation effects, albeit only for a parallel shear flow. The method is dependent on the location $z_{0}$ chosen for analysis. Moreover the Lilley-Goldstein equation suffers from a well-known problem of instability when time-domain calculations are attempted, as in the present study. In agreement with Cheung et al.(2007), we are only able to obtain stable solutions when the base profile location is chosen to be sufficiently far downstream that the particular frequency under consideration is stable.

An alternative method of solving equation (1) is possible, at least for the $S_{z z}$ source term, based on the jump relations for the Lilley-Goldstein equation. In this approach (see Appendix B of Sandham et al., 2006) equation (1) is integrated across a shear layer, leading to relations between the jumps in $p$ and $\partial p / \partial y$ and the source terms $f$ and $q$ defined by equation (2). The jump relations are given by

$$
\Delta p=\bar{\rho} \hat{f}_{y}
$$

and

$$
\Delta p^{\prime}=\bar{\rho}\left(\frac{\partial \hat{f}_{x}}{\partial x}-\frac{D \hat{q}}{D t}\right),
$$

where $\Delta p$ and $\Delta p^{\prime}$ are the jumps in pressure and pressure derivative and source terms are concentrated according to $f=\hat{f} \delta\left(y-y_{0}\right)$ where $y_{0}$ is the location of the inflection point in the shear layer. The basic idea is then to solve the problem for $y \geq y_{0}$ and $y \leq y_{0}$ in a coupled manner with boundary conditions applied at $y_{0}$ and hence avoiding an inflection point in the field of computation (and so hoping to avoid the instability in the Lilley-Goldstein equation).

For the present round jet configuration some results of this approach are shown in Figure 8. The $S_{z z}$ source has been concentrated on $r=r_{0}$, where $r_{0}$ is the location of the inflection point at the particular 
streamwise location $z_{0}$ chosen for study, by computing

$$
\hat{f}_{z}=\frac{1}{r_{0}} \int_{0}^{L} r f_{z} \mathrm{~d} r
$$

and the computation is carried out applying a boundary condition for $\partial p / \partial r$. Figure $8($ a) shows the results of using this approach, but setting $\bar{V}_{z}=0$ and $\bar{\rho}=1$ throughout, effectively solving equation (3) by a different approach. Figure 8(a) compares favourably with the $S_{z z}$ driven acoustic field on figure 3(a), showing that the method preserves the important properties of the source. Figures $8(\mathrm{~b})$ and $8(\mathrm{c})$ use velocity and density profiles at $z_{0}=5$ and $z_{0}=15$ respectively. These calculations were run to a longer time and in a larger domain than those presented earlier, to check for any instability in the method. No instability is found and it appears that with this method any $z_{0}$ can be chosen and gives stable results. Figure 8(b) shows a strong near field response for $z_{0}=5$ and stronger radiation in the downstream arc compared to figure 8 (a). With $z_{0}=15$ (figure $8(\mathrm{c})$ ) there is more evidence of refraction, with the peak sound radiation at $20^{\circ}$ to the downstream direction (based on a source origin at $z=7$ ).

Attempts to apply the same method to the other sources have so far been frustrated by the difficulty of devising an appropriate form of (18) for sources that are not very coherent in space and by the appearance of an unconstrained algebraic growth of the near field when forcing is applied via a pressure boundary condition, as required by equation (16) for several source terms.

\section{Results for a variable base flow}

Up until now all the PSE results have been based on the fixed base flow given by equations (11-14). An alternative approach is possible in which nonlinear interactions of PSE modes are used to update the mean flow. In the current implementation the boundary layer equations are solved by a downstream marching procedure alongside the PSE. As well as viscous stresses there are Reynolds stress terms due to mode interactions, for example the self-interaction of a PSE mode at a particular frequency will give a difference frequency of zero and hence a mean flow modification. This means that the shear layer will thicken as a result of each mode growth. Eventually the mean flow grows sufficiently for the mode to saturate. This type of calculation was demonstrated first by Morris et al (1990) for a spatially developing mixing layer. In that work eigensolutions of the Orr-Sommerfeld equation were used to provide the growth rate of similarity profiles for velocity and temperature. A valuable result was to provide a theoretical underpinning for the known qualitative connection between mixing layer spreading rate and the growth rate of the most unstable instability mode. Follow up work by Viswanathan \& Morris (1992) extended the approach to axisymmetric shear layers, while Liou \& Morris relaxed the requirement for prescribed shape functions.

We employ a method in which the profiles defined by equations (11-14) are used as a template for the flow. For each step downstream the boundary layer equations are solved to give a provisional updated streamwise velocity profile $V_{z}^{*}(r)$. The parameters $\delta$ and $a$ that give a minimum least squares error to the provisional profile are then found and these parameters define the profile $\bar{V}_{z}(r)$ at the new streamwise location. This method has been found to be more robust than when the provisional profile is used by itself. In particular, unstable narrow shear layers that might cause the simulation to terminate are prevented from developing.

Figure 9 shows the result from a calculation where two axisymmetric modes with frequencies of 3.4 and 2.2 are computed with PSE and the mean flow evolves by both laminar spreading and the action of the Reynolds stresses. The modes were normalized at $z=0$ with their integrated intensity $q^{2}$ and then applied with an amplitude of $10^{-4}$ at $z=0$. No attempt was made to fix the relative phases. Figure 9 (a) shows $\delta$ from the simulation, compared with the prescribed $\delta$ used in earlier calculations and Figure 9(b) shows the corresponding $n$-factors. Initially the flow spreads according to laminar diffusion. The higher frequency mode grows most strongly at $z=3$ and reaches a maximum amplitude at $z=3.5$. It then decays, but in the process has doubled the shear layer thickness. The second maximum in $\delta$ occurs at $z=8$ corresponding to the axial location where the lower frequency mode saturates. Thereafter the shear layer thickness decreases slowly since there are no lower frequencies present that could lead to further growth.

The sound produced by the interaction is shown on figure 10 with contours of $\pi$ computed from a solution of the second order wave equation (3) for comparison with figure 3(a). The radiation pattern due to the four sources is similar. The strongest radiation again comes from $S_{z z}$, followed by $S_{r r}$ and only very weak radiation from $S_{r z}$ and $S_{z r}$. There are visible differences however, notably the additional lobe of radiation in the $S_{r r}$ source at $90^{\circ}$ to the jet axis. 
(a)

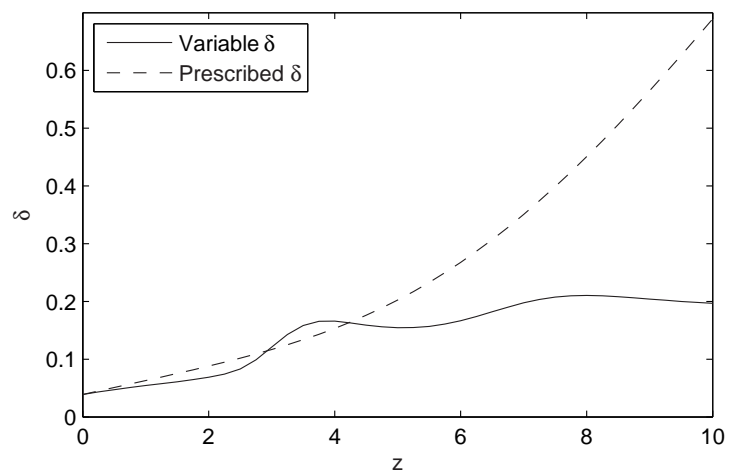

(b)

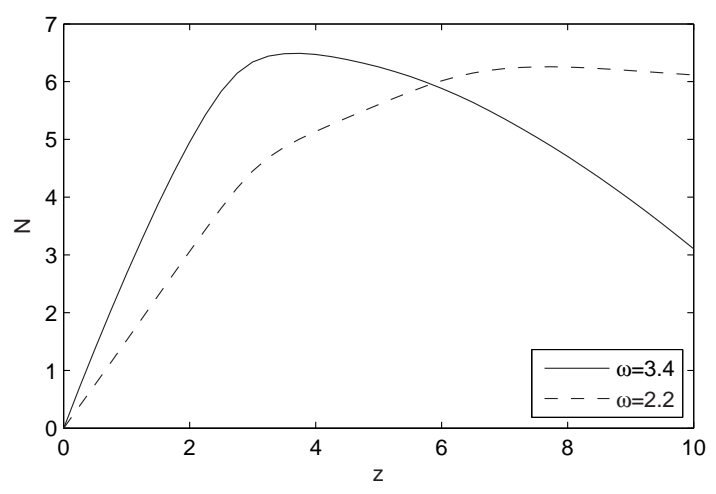

Figure 9. Growth rate of jet: (a) $\delta$ obtained from PSE, driven with two frequencies, compared with the empirical relation (11-13), (b) growth $(N-)$ factors for each mode
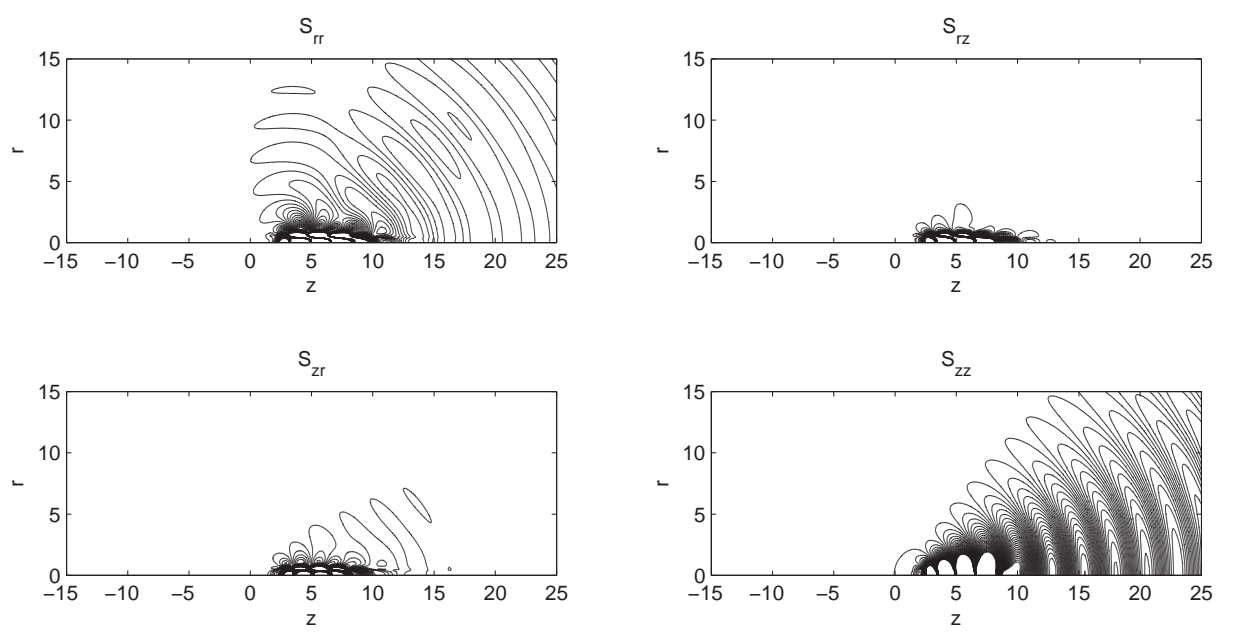

Figure 10. Contours of pressure from solution of wave equation driven by the difference mode interaction of axisymmetric modes for the variable base flow case. Source terms are injected for $S_{r r}, S_{r z}, S_{z r}$ and $S_{z z}$ as shown in the sub-figure titles. 


\section{Conclusions}

A nonlinear interaction model has been used to investigate the radiation patterns resulting from nonlinear interactions between instability modes. For any frequency and azimuthal wavenumber combination the radiated sound resulting from up to nine possible source components can be extracted. It is found that difference mode interactions are more important than sum modes. Particularly important difference-mode sources are $S_{z z}$ and $S_{r r}$ from axisymmetric interactions and the combination of $S_{r r}$ and $S_{\theta \theta}$ for the interaction between two helical modes.

The effect of the wave equation was studied by comparing results from the simple second-order acoustic wave equation with results from the Lilley-Goldstein third-order system for the $S_{z z}$ source. The higher-order wave equation leads to more radiation into the forward arc. The results with the Lilley-Goldstein equation are sensitive to the choice of mean flow.

A modified approach, where the mean flow is determined by the growing instabilities (via the Reynolds stresses) was compared with the approach of a prescribed base flow for a case with two axisymmetric modes. The method appears to be realistic in capturing the growth, saturation and decay of instability modes and the corresponding mean flow modifications. The radiation pattern is similar to that computed with the fixed base flow. Overall the simplified mode interaction approach appears to provide useful insight into sound generation mechanisms.

\section{Acknowledgements}

This work is supported by EPSRC, grant number EP/E032028/1.

\section{References}

\footnotetext{
${ }^{1}$ Cheung, L.C., Bodony, D.J. and Lele, S.K. 2007 Noise radiation predictions from jet instability waves using a hybrid nonlinear PSE-acoustic analogy approach. AIAA 2007-3638.

${ }^{2}$ Freund, J.B. 2001 Noise sources in a low-Reynolds-number turbulent jet at Mach 0.9. J. Fluid Mech. 438, 277-305.

${ }^{3}$ Herbert, Th. 1997 Parabolized stability equations. Annual Review of Fluid Mechanics 29, 245-283.

${ }^{4}$ Hein, S. 2005 Nonlinear nonlocal transition analysis. DLR-Forschungsbericht 2005-10.

${ }^{5}$ Goldstein, M.E. 2001 An exact form of Lilley's equation with a velocity quadrupole/temperature dipole source term. J. Fluid Mech. 443, 231-236.

${ }^{6}$ Laufer, J. and Yen, T.-C. 1983 Noise generation by a low-Mach-number jet. J. Fluid Mech. 134, 1-31.

${ }^{7}$ Lewis, H.R. and Bellan, P.M. 1990 Physical constraints on the coefficients of fourier exansions in cylindrical coordinates. Journal of Maths and Physics, 31:11, 2592-2596.

${ }^{8}$ Lighthill, M.J. 1952 On sound generated aerodynamically: I. General theory. Proc. R. Soc. Lond. A 222, 564-587.

${ }^{9}$ Lilley, G.M. 1974 On the noise from jets. AGARD CP-131, 13.1-13.12.

${ }^{10}$ Liou, W.W. and Morris, P.J. Weakly nonlinear models for turbulent mixingm in a plane mixing layer. Physics of Fluids A-Fluid Dynamics 4(12), 2798-2808.

${ }^{11}$ Morfey, C.L. and Wright, M.C.M. 2007 Extensions of Lighthill's acoustic analogy with application to computational aeroacoustics. Proc. Royal Society A 463(2085), 2101-2127.

${ }^{12}$ Morris, P.J., Giridharan, M.G. and Lilley, G.M. 1990 On the turbulent mixing of compresible free shear layers. Proc. R. Soc. Lond. A 431, 219-243.

${ }^{13}$ Sandham, N.D., Morfey, C.L. and Hu, Z.W. 2006a Nonlinear mechanisms of sound generation in a perturbed parallel jet flow Journal of Fluid Mechanics, 565, 1-23.

${ }^{14}$ Sandham, N.D., Morfey, C.L. and Hu, Z.W. 2006 Sound radiation from exponentially growing and decaying surface waves. Journal of Sound and Vibration, 294 (1-2), 355-361.

${ }^{15}$ Sandham, N.D. and Salgado, A.M. 2008 Nonlinear interaction model of subsonic jet noise. Accepted for publication in Phil. Trans.

${ }^{16}$ Stromberg, J.L., McLaughlin, D.K. and Troutt, T.R. 1980 Flow field and acoustic properties of a Mach number 0.9 jet at a low Reynolds number. Journal of Sound and Vibration, 72 (2), 159-176.

${ }^{17}$ Suzuki, T. and Colonius, T. 2006 Instability waves in a subsonic jet detected using a near-field phased microphone array. J. Fluid Mech. 565, 197-226.

${ }^{18}$ Tam, C.K.W. and Burton, D.E. 1984 Sound radiated by instability waves of a supersonic flows. Part 2. Axisymmetric jets. J. Fluid Mech. 138, 273-295.

${ }^{19}$ Tam, C.K.W. and Morris, P.J. 1980 The radiation of sound by the instability waves on a compressible plane turbulent shear layer. J. Fluid Mech. 98(2), 349-381.

${ }^{20}$ Viswanathan, K. and Morris, P.J. 1992 Predictions of turbulent mixing in axisymmetrical compressible shear layers. AIA A Journal 30(6), 1529-1536.
} 